\title{
A 475 years-old founder effect involving IL12RB1: A highly prevalent mutation conferring Mendelian Susceptibility to Mycobacterial Diseases in European descendants
}

\author{
J. Yancoski ${ }^{a}$, C. Rocco ${ }^{a}$, A. Bernasconi ${ }^{a}$, M. Oleastro $^{a}$, L. Bezrodnik $^{b}$, C. Vrátnica ${ }^{c}$, \\ F. Haerynck ${ }^{\mathrm{d}}$, S.D. Rosenzweig ${ }^{\mathrm{a}, *}$ \\ ${ }^{a}$ Hospital Nacional de Pediatría J. P. Garrahan, Buenos Aires, Argentina \\ ${ }^{\mathrm{b}}$ Hospital de Niños Ricardo Gutierrez, Buenos Aires, Argentina \\ ${ }^{\mathrm{c}}$ Hospital de Niños Juan Pablo II, Corrientes, Argentina \\ ${ }^{\mathrm{d}}$ Ghent University Hospital, Ghent, Belgium
}

\section{A R T I C L E I N F O}

\section{Article history:}

Received 11 December 2008

Received in revised form 13 February 2009

Accepted 16 February 2009

Available online 9 March 2009

\section{Keywords:}

Hot spot

Salmonella

Bacillus Calmette Guerin

Interferon gamma

Linkage disequilibrium

\begin{abstract}
A B S T R A C T
Mutations in IFNGR1, IFNGR2, IL12RB1, IL12B, STAT1 and NEMO result in a common clinical phenotype known as Mendelian Susceptibility to Mycobacterial Diseases (MSMD). Interleukin-12 receptor $\beta 1$ (IL$12 \mathrm{R} \beta 1$ ) deficiency is the most common genetic etiology for MSMD. Known mutations affecting IL12RB1 are recessively inherited and are associated with null response to both IL-12 and IL-23. Mutation IL12RB1 1623_1624delinsTT was originally described in 5 families from European origin ( 2 from Germany; 1 from Cyprus, France and Belgium). Interestingly, this same mutation was found in an unexpectedly high prevalence among IL-12R $\beta 1$ deficient patients in Argentina: 5-out-of-6 individuals born to unrelated families carried this particular change. To determine whether mutation 1623_1624delinsTT represents a DNA mutational hotspot or a founder effect, 34 polymorphic markers internal or proximal to IL12RB1 were studied in the Argentinean and the Belgian patients. A common haplotype spanning $1.45-3.51 \mathrm{Mb}$ was shared by all chromosomes carrying mutation 1623_1624delinsTT, and was not detected on 100 control chromosomes. Applying a modified likelihood-based method the age of the most recent common ancestor carrying mutation 1623_1624delinsTT was estimated in 475 years (95\% CI, 175-1275), which is the time when the Spaniards initiated the colonization of the Americas. Mutation 1623_1624delinsTT represents the first founder effect described on IL-12R $\beta 1$, the most frequently affected gene in MSMD, and affecting patients with European ancestors. The reason(s) behind the persistency of this mutation across multiple generations, its relative high prevalence, and any potential selective advantage are yet to be established.
\end{abstract}

Published by Elsevier B.V.

\section{Introduction}

With more than 100 affected patients worldwide, IL-12R $\beta 1$ deficiency is the most common genetic etiology for Mendelian susceptibility to Mycobacterial Disease (MSMD). Interleukin-12 receptor $\beta 1$ deficiency causes an illness characterized by a selective susceptibility to poorly pathogenic mycobacteria, and nontyphoid Salmonella species attesting to the importance of IL-12R in the host defense against intracellular pathogens (Altare et al., 1998; de Jong et al., 1998; Fieschi et al., 2003; van de Vosse and Ottenhoff, 2006; Filipe-Santos et al., 2006; Rosenzweig et al., 2006).

\footnotetext{
* Corresponding author at: Servicio de Inmunología, Hospital Nacional de Pediatría J. P. Garrahan, Combate de los Pozos 1881, Cdad. de Buenos Aires (1245), Argentina. Tel.: +54 9115408 2641; fax: +54 1143084076 .

E-mail address: srosenzweig@garrahan.gov.ar (S.D. Rosenzweig).
}

Mutations on the IL12RB1 gene are associated with impaired response to IL-12 and IL-23 (Altare et al., 1998; de Jong et al., 1998; Hoeve et al., 2003). With only one exception, all IL-12R $\beta 1$ deficient patients described displayed no detectable IL-12R $\beta 1$ on their cell surface due to mutations that either interrupt the open reading frame (nonsense and frameshift mutations) or disrupt folding of the protein (missense mutations) (Altare et al., 1998; de Jong et al., 1998; Fieschi et al., 2003; Fieschi et al., 2004; van de Vosse and Ottenhoff, 2006; Filipe-Santos et al., 2006). Complex mutation 1623_1624delinsTT (GC deletion, TT insertion) results in a silent point mutation (V541 V) and the generation of a stop codon (Q542X) in IL12RB. This particular change had been previously described in 5 families from European origin ( 2 from Germany; and 1 from Cyprus, France and Belgium) (Fieschi et al., 2003; Haerynck et al., 2008). Unexpectedly, mutation 1623_1624delinsTT was found in 5-out-of-6 Argentinean IL-12R $\beta 1$ deficient patients ( 3 homozygous and 2 heterozygous) from 6 unrelated families. 
The finding of identical mutations in apparently unrelated patients from different regions in the planet suggested that recurrent mutations could arise (mutational hot spot). Alternatively, it was possible that despite the geographic separation the families shared a common ancestry (mutational founder effect). Herein, these two alternative hypotheses are explored.

\section{Patients, materials and methods}

\subsection{Patient cohort}

Six patients with complete IL-12R $\beta 1$ deficiency and born to 6 unrelated non-consanguineous Argentinean families were referred to the Hospital Nacional de Pediatría J. P. Garrahan in Buenos Aires, Argentina and evaluated for this study. All the patients had been assessed for IFN $\gamma /$ IL-12/IL-23 pathway integrity after they developed disseminated mycobacterial infections early on life. All the patients developed disseminated $M$. bovis-BCG infection after routine BCG vaccination. None of the patients expressed IL-12R $\beta 1$ on the surface of their T cells or NK cells as determined by flow cytometry and their lymphocytes did not respond to IL-12 stimulation ex vivo. All the patients were screened for mutations on $I L-12 R \beta 1$ by PCR amplification of genomic DNA (17 exons and exon/intron boundaries), followed by direct sequencing of the PCR products. Among this cohort, mutation 1623_1624delinsTT was found in 5 affected individuals, all of them born to European ancestors. Three patients were homozygous and 2 heterozygous for this trait. Another patient, Belgium-born and heterozygous for the same mutation, was also included in this study.

The patients' ancestry was established by direct questioning for at least 4 generations in each affected family with no evidence of consanguinity. However, encrypted relatedness could not be ruled out completely.

Thirty-four polymorphic sites on chromosome 19 were analyzed in these patients: 21 intragenic IL12RB1 (15 exonic, 6 intronic) and 13 extrageneic. All markers were tested on gDNA. In addition, IL-12R $\beta 1$ mRNA was extracted, cDNA converted, PCR amplified, subcloned and sequenced in heterozygous patients for individual-allele IL12RB1-exonic polymorphic markers determination.

Genomic DNA was obtained by saline extraction from PBMCs. Single nucleotide polymorphisms' genotyping was performed with DYEnamic ET Terminator Cycle Sequencing Kit (Amersham Biosciences, Little Chalfont Buckinghamshire, UK) from PCR products obtained using specific primers (Appendix) and Taq polymerase (Invitrogen, Carlsbad, CA). Messenger RNA was extracted from PBMCs of 1623_1624delinsTT heterozygous patients according to the manufacturer's instructions (Trizol, Invitrogen, Invitrogen, Carlsbad, CA). Messenger RNA was reverse transcribed to cDNA using Oligo-dT (Invitrogen, Carlsbad, CA) and reverse transcriptase (Invitrogen, Carlsbad, CA) following producers' protocols. Interleukin-12 receptor $\beta 1$ cDNA was amplified with Taq polymerase and specific primers. The obtained PCR product was cloned into a TOPO TA vector (TOPO TA Cloning kit, Invitrogen, Carlsbad, CA) and later used to transform TOP10 One Shot Chemically Competent cells (Invitrogen, Carlsbad, CA). Transformed bacteria were seeded on Luria Bertani (LB)-XGalAmpicillin (Amp) plates, efficiently-transformed bacteria were grown in LB-Amp media, plasmidic DNA was extracted (Wizard Plus SV Minipreps, Promega, Madison, WI), sequenced on an ABI PRISM 3130 Genetic Analyzer, and analyzed using Gene Scan software (Applied Biosystem). Microsatellites were PCR-amplified with fluorescent-labeled specific F primers, run on an ABI PRISM 3130 Genetic Analyzer, and analyzed using the Gene Scan software.

\subsection{Control population}

Genomic DNA was extracted from 50 unrelated healthy Argentinean blood donors of European-descent (100 chromosomes). Samples were screened for SNPs G451C and A705G by restriction fragment length polymorphism (RFLP) analysis and/or direct sequencing. For SNP A705G allele frequency determination, exon 7 IL12RB1 was PCR-amplified with specific primers, the obtained product was PvuII-digested according to manufacturer's recommendations (New England Biolabs, Ipswish, MA), resolved in $2 \%$ agarose gels, stained with ethidium bromide and analyzed under UV light. Digestion with PvuII in the presence of allele A705 generates 2 fragments of 107 and $91 \mathrm{bp}$ and ruled out the presence of the mutated haplotype. For G451C allele frequency determination, exon 4 IL12R $\beta 1$ was PCR-amplified with specific primers, the obtained product was digested with restriction enzyme HphI (New England Biolabs, Ipswish, MA), PAGE resolved in NOVEX 20\% TBE gels (Invitrogen, Carlsbad, CA), stained with ethidium bromide and analyzed under UV light. Digestion with $\mathrm{HphI}$ generates a set of four fragments $(320,172,112$ and $36 \mathrm{bp})$ in the presence of allele $451 \mathrm{C}$, while a different set of fragments $(284,226,94$ and $36 \mathrm{bp})$ is observed in the presence of allele G451. Homozygosity for G451 ruled out the presence of the mutated haplotype. Six normal controls ( 12 chromosomes) were tested by direct sequencing of the entire coding region of IL12R $\beta 1$ gene.

All patients and controls gave written consent for DNA/RNA extraction and analysis. Primer sequences are shown in the Appendix A, Table A1.

\subsection{Mutation 1623_1624delinsTT age estimation}

In order to estimate the age to the most recent common ancestor of haplotypes carrying the 1623_1624delinsTT mutation, a previously described likelihood method was modified and applied on a set of six recombination markers located at variant distances on both sides of the disease mutation (Genin et al., 2004). The approximation was introduced by Genin and collaborators as an interval censored survival analysis in which the mutation locus, denoted as $\Delta$, is the starting point, the genetic distance is the time scale and the occurrence of recombination is the event. The model takes the following basic assumptions:

- monophyletic origin of the disease mutation

- independence of each sampled chromosome and equal time to a common ancestor

- independent recombination rates at each side of $\Delta$

- negligible probability of double recombination

Given $M_{1}, \ldots, M_{x}, \ldots, M_{\mathrm{k}} k$ ordered markers typed on one side of $\Delta$, let $\theta_{1}, \ldots, \theta_{x}, \ldots, \theta_{k}$ be the recombination fractions from $\Delta$ (for convenience, $\theta_{0}=0$ and $\left.\theta_{k+1}=1\right)$. Define $S(x)$, the probability that no recombination took place during $n$ generations between $\Delta$ and $M_{x}$ as

$S(x)=\left(1-\theta_{x}\right)^{n}$.

Therefore, the probability of recombination in the $x$ th intervalbetween $M_{x-1}$ and $M_{x}$-namely $f(x)$, is

$f(x)=S(x-1)-S(x)$.

Taking advantage of this construction, missing information on $M_{x-1}$ for any sampled chromosome could be handled by considering the most distant marker from $\Delta$ without recombination $\left(M_{x-2}, M_{x-3}\right.$, etc). However, as the intervals include more missing markers the assumption of negligible double recombination becomes less sustainable, and further formulations would be needed. Under the described model, the likelihood function is 
constructed independently for each side of $\Delta$ and for a sample of $N$ chromosomes two situations should be noticed. In the first one, every chromosome shows evidence of recombination to one side of $\Delta$. In this case, there are two groups of haplotypes. In one group, denoted as $G_{1}, y$ chromosomes $(2 \leq y \leq N)$ share all their alleles for markers $M_{1}, \ldots M_{k-1}$ and have a different allele at $M_{k}$. For the $G_{1}$ group, the likelihood is written

$L_{G_{1}}(n)=f(k)^{y}+y S(k) f(k)^{y-1}$,

taking into account the uncertainty on which the ancestral allele is. In the remaining $N-y$ chromosomes, $G_{2}$, a recombination occurred on the interval $x_{i}(1 \leq i \leq N-y)$ closer to $\Delta\left(x_{i} \leq k\right)$. For $G_{2}$ the likelihood is written

$L_{G_{2}}(n)=\prod_{i \in G_{2}} f\left(x_{i}\right)$.

A less desirable, unless possible, scenario is found when there is no recombination before $M_{k}$. In other words, a set of $z$ chromosomes, group $G_{3}$, present the same alleles for $M_{1}$ to $M_{k}$. Accordingly, likelihood is

$L_{G_{3}}(n)=S(k)^{z}$.

Note that when the $G_{3}$ group is not empty then $G_{1}$ is. Finally, chromosomes with missing data for markers $M_{a}, \ldots, M_{k}(1 \leq a \leq k)$ and without evidence of recombination for markers $M_{1}, \ldots, M_{\mathrm{a}-1}$ conform $G_{4}$, with likelihood

$L_{G_{4}}(n)=\prod_{j \in G_{4}} S\left(x_{j}\right)$

where $x_{j}<k$ is the interval to the last marker with available information and no recombination.

In conclusion, the likelihood for $n$ generations on the studied side is

$\begin{aligned} L_{\text {side }}(n)= & {\left[I_{G_{1} \neq \varnothing} L_{G_{1}}(n)+I_{G_{3} \neq \varnothing} L_{G_{3}}(n)+\left(1-I_{G_{1} \neq \varnothing}\right.\right.} \\ & \left.-I_{\left.G_{3} \neq \varnothing\right)}\right) L_{G_{2}}(n) L_{G_{4}}(n),\end{aligned}$

where $I_{G_{\alpha} \neq \varnothing}$ equals 0 if group $\alpha$ is empty and 1 if it is not. The final likelihood, $L(n)$, is the product of the two side likelihoods. Point estimation of generation number $n$ is obtained at $L(n)$ maximum.

Equations (1)-(5) hold without ambiguity if the presence or absence of a recombination event within intervals is known. However, they do not account for mutation, resulting in a false positive recombination signal, or low allele diversity, resulting in a false negative recombination signal. The adjustments introduced to deal with this ambiguity are described in the Appendix. Further calculation for 1623_1624delinsTT included both corrections.

Computation of 95\% confidence intervals (CI) for an estimated generation number was based on Bayesian principles. Briefly, 95\% $\mathrm{CI}$ is given by all $n$ which satisfies:

$0.025 \leq \frac{\sum_{u=1}^{n} L(u)}{\sum_{v=1}^{V} L(v)} \leq 0.975$,

where $V$ is a large generation number (e.g. $10^{4}$ ).

Algorithms were programmed on $\mathrm{R}$ version 2.6.0, a free software environment. Scripts are available upon request. Likelihood corrections for mutation and allele frequency calculation are described in the Appendix.

\subsection{Extragenic markers recombination fraction estimation}

The preceding theory is based on a priori knowledge on recombination fractions $\theta_{1}, \ldots, \theta_{k}$. Thereafter, recombination rates for the intervals among the selected extragenic markers at both sides of 1623_1624 delGCinsTT were estimated by means of a robust linear regression, carried out with published data on 37 neighboring polymorphic sites incorporated into the Marshfield linkage map (Fig. 2). Linkage (Kosambi centiMorgans, cM) was inferred for D19S1037, CRLF1, D19S895, SFRS14, TSSK6 and D19S215 based on sequence position on chromosome 19 (mega base pairs, Mb). Calculated fractions are presented in Table 1 . The result represents an average of $1.16 \mathrm{cM}$ per $\mathrm{Mb}$ across the entire region.

\section{Results}

\subsection{Mutation 1623_1624delinsTT haplotyping}

All patients carrying mutation 1623_1624delinsTT in homozygous or heterozygous state shared a common haplotype on the mutated allele of chromosome 19, which included the complete IL12RB1 gene (Table 1). Thirty-four polymorphic sites on chromosome 19 were analyzed: 21 intragenic IL12RB1 (15 exonic, 6 intronic) and 13 extrageneic. Of the 34 markers analyzed, 32 were included on the mutated haplotype (from C72A to TSSK6:T989C; distance between markers, $1.45 \mathrm{Mb}$ ). Markers D19S1037 and D19S215, which showed variations in homozygous and heterozygous patients, flanked and defined the mutated haplotype (distance between markers, $3.51 \mathrm{Mb}$ ).

None of the 100 control chromosomes analyzed carried the mutated haplotype above described. Control chromosomes were screened for SNPs G451C and A705G (variants 451C and 705G on the mutated allele) by restriction fragments length polymorphism (RFLP) analysis (Fig. 1). Eighty-eight percent of the tested samples were excluded as carrying the mutated haplotype by this method; the remaining $12 \%$ were ruled out by IL12RB1 gDNA direct sequencing. Since the mutated haplotype was not found in any of the 100 control chromosomes tested, its frequency remains $\leq 0.01 \%$ in the general population. Therefore, the probability that mutation 1623_1624delinsTT have occurred by chance sharing the same set of polymorphic markers in the 9 affected alleles is not larger than the probability of randomly sampling nine times in a row the mutated haplotype, namely $(0.01)^{9}=10^{-18}$. Together these data strongly suggested that the 1623_1624delinsTT mutated allele originated from a single founder.

\subsection{Mutation 1623_1624delinsTT age calculation}

The most likely age for mutation 1623_1624delinsTT was 19 generations, with a $95 \%$ Confidence Interval (CI) of 7-51. Considering 25 years for each generation, this represents 475 years $(95 \% \mathrm{CI}, 175-$ 1275) (Fig. 2). Estimation of the mutation age was calculated from 6 extragenic recombination markers applying a modified likelihoodmethod based on that originally described by Genin et al., 2004. Variations on microsatellites D19S1037, D19S895, and D19S215, and 10 SNPs distributed along genes CRLF1 (3 SNPs), SFRS14 (5 SNPs) and TSSK6 (2 SNPs) were tested. The 10 SNPs were considered as 3 independent loci-one for each gene-assuming null recombination frequencies within each gene. Mutations rates and allele frequencies were taken into account according to previous reports (Weber and Wong, 1993; Nachman and Crowell, 2000). Mutation rates were set to $5.6 \times 10^{-4}, 2.1 \times 10^{-3}$ and $2.55 \times 10^{-8}$ for dinucleotide tandem repeats, tetranucleotide tandem repeats, and SNPs, respectively, as shown in Section 2.

To assess the contribution of the assumed mutation frequencies to the estimated time of the founder mutation, the same calculations were carried out for varying mutation rates. Assumption that the rate of mutations was one order (10 times) higher resulted in an estimation of 12 generations ( $95 \% \mathrm{CI}, 4-30$ ), while if the rate was assumed to be one order lower, the estimated mutation age would be 21 generations (95\% CI, 7-55). Of note, these modifications yielded a projected outcome encompassed by the initial confidence 


\begin{tabular}{|c|c|c|c|c|c|c|c|c|c|c|c|}
\hline Marker & rs/UniSTS & Location & PD (Mb) & $\mathbf{A F}$ & RF & P1 & P2 & P3 & P4 & P5 & P6 \\
\hline D19S1037 & UniSTS:38487 & Extragenic & 0,094 & 0.143 & 0.138 & $1 / 2$ & $2 / 3$ & ND & $2 / 4$ & ND & $1 / 4$ \\
\hline C $72 \mathrm{~A}$ & rs17884651 & ILI2RBI ex. & & 1 & im & C & C & $\mathrm{C}$ & C & $\mathrm{C}$ & C \\
\hline C $202 \mathrm{~T}$ & rs17887176 & ILI2RBI ex. & & 1 & im & $\mathrm{C}$ & C & C & C & $\mathrm{C}$ & $\mathrm{C}$ \\
\hline G $451 \mathrm{C}$ & rs11086087 & $I L 12 R B I$ ex. & & 0.087 & im & $\mathrm{C}$ & C & $\mathrm{C}$ & C & $\mathrm{C} / \mathrm{G}$ & $\mathrm{C} / \mathrm{G}$ \\
\hline G $531 \mathrm{~A}$ & rs11575926 & ILI2BBI ex. & & 0.804 & im & G & G & G & G & G/A & G \\
\hline A $705 \mathrm{G}$ & rs11575934 & $I L 12 R B I$ ex. & & 0.304 & im & G & G & G & G/A & G/A & G \\
\hline C 748 T & rs17852635 & $I L 12 R B I$ ex. & & 0.295 & im & $\mathbf{T}$ & $\mathbf{T}$ & $\mathbf{T}$ & $\mathrm{T} / \mathrm{C}$ & $\mathrm{T} / \mathrm{C}$ & $\mathbf{T}$ \\
\hline C $1081 \mathrm{~A}$ & rs17884957 & $I L 12 R B I$ ex. & & 1 & im & $\mathrm{C}$ & $\mathrm{C}$ & $\mathrm{C}$ & $\mathrm{C}$ & $\mathrm{C}$ & C \\
\hline T $1158 \mathrm{C}$ & rs375947 & $I L I 2 R B I \mathrm{ex}$. & & 0.304 & im & $\mathrm{C}$ & C & C & $\mathrm{C} / \mathrm{T}$ & $\mathrm{C} / \mathrm{G}$ & C \\
\hline G $1196 \mathrm{C}$ & rs401502 & $I L I 2 R B I$ ex. & & 0.696 & im & $\mathrm{C}$ & C & $\mathrm{C}$ & $\mathrm{C} / \mathrm{G}$ & $\mathrm{C} / \mathrm{G}$ & $\mathrm{C} / \mathrm{G}$ \\
\hline C $1213 \mathrm{~T}$ & rs17882216 & $I L 12 R B I$ ex. & & 1 & im & $\mathrm{C}$ & C & C & $\mathrm{C}$ & $\mathrm{C}$ & $\mathrm{C}$ \\
\hline C $1306 \mathrm{~T}$ & rs376271 & ILI2RBI ex. & & ND & im & C & C & C & C & C & C \\
\hline $\mathrm{C} 1376 \mathrm{~T}$ & & $I L 12 R B 1$ ex. & & ND & im & C & C & C & C & C & C \\
\hline 1623_1624delinsTT & & Mutation & & & & Homoz & Homoz & Homoz & Heteroz & Heteroz & Heteroz \\
\hline $\mathrm{C} 108530 \mathrm{~T}$ & & ILI2RBI int. & & ND & $\mathrm{im}$ & C & C & $\mathrm{C}$ & C & $\mathrm{C}$ & C \\
\hline C $108571 \mathrm{~T}$ & & ILI2RBI int. & & ND & im & $\mathbf{T}$ & $\mathbf{T}$ & $\mathbf{T}$ & $\mathbf{T}$ & $\mathrm{T} / \mathrm{C}$ & $\mathrm{T} / \mathrm{C}$ \\
\hline G $108616 \mathrm{~A}$ & & $I L I 2 R B I$ int. & & ND & im & $\mathbf{A}$ & $\mathbf{A}$ & $\mathbf{A}$ & A & $\mathrm{A} / \mathrm{G}$ & $\mathrm{A} / \mathrm{G}$ \\
\hline T $108661 \mathrm{C}$ & & ILI2RBI int. & & ND & im & $\mathrm{C}$ & C & $\mathrm{C}$ & C & $\mathrm{C} / \mathrm{T}$ & $\mathrm{C}$ \\
\hline G $1637 \mathrm{~A}$ & rs 11575935 & $I L 12 R B I$ ex. & & ND & im & G & G & G & G & G & G \\
\hline C $1783 \mathrm{~T}$ & rs17885102 & $I L 12 R B I$ ex. & & 1 & im & $\mathrm{C}$ & C & C & C & $\mathrm{C}$ & C \\
\hline G $1845 C$ & & $I L 12 R B I$ ex. & & ND & im & G & G & G & G & G & G \\
\hline G rh66781 A & UniSTS:47762 & $I L 12 R B I$ int. & & ND & im & G & G & G & G & G & G \\
\hline 5C SHGC156161 6C & UniSTS: 185287 & ILI2RBI int. & & ND & im & $6 \mathrm{C}$ & $6 \mathrm{C}$ & $6 \mathrm{C}$ & $6 \mathrm{C}$ & $6 \mathrm{C} / 5 \mathrm{C}$ & $6 \mathrm{C}$ \\
\hline CRLF1:G6489A & rs 2238647 & Extragenic & 0,506 & 0.783 & 0.596 & G & $\mathbf{G}$ & G & G & G & G \\
\hline CRLF1: G6560- & rs34603196 & Extragenic & 0,506 & ND & 0.596 & G & G & G & G & G & G \\
\hline CRLF1:C6579T & rs7259478 & Extragenic & 0,506 & 1 & 0.596 & C & C & C & C & C & C \\
\hline D19S895 & UniSTS:79811 & Extragenic & 0,555 & 0.376 & 0.645 & $1 / 1$ & $1 / 1$ & $1 / 1$ & $1 / 2$ & $1 / 1$ & $1 / 3$ \\
\hline SFRS14:C274T & rs35401849 & Extragenic & 0,904 & 0.987 & 1.088 & $\mathrm{C}$ & C & $\mathrm{C}$ & C & C & C \\
\hline SFRS14:A305G & rs 10404860 & Extragenic & 0,904 & 1 & 1.088 & A & A & A & A & A & A \\
\hline SFRS14:G606T & rs12459416 & Extragenic & 0,904 & 1 & 1.088 & G & G & G & G & G & G \\
\hline SFRS14T1102C & rs34471092 & Extragenic & 0,904 & 0.986 & 1.088 & $\mathbf{T}$ & T & $\mathbf{T}$ & $\mathbf{T}$ & $T$ & $\mathbf{T}$ \\
\hline SFRS14:C1344T & rs4808907 & Extragenic & 0,904 & ND & 1.088 & $\mathrm{C}$ & $\mathrm{C}$ & $\mathrm{C}$ & $\mathrm{C}$ & $\mathrm{C}$ & $\mathrm{C}$ \\
\hline TSSK6:A350G & rs7250893 & Extragenic & 1,427 & 0.562 & 1.653 & $\mathbf{A}$ & A & $\mathbf{A}$ & $\mathbf{A}$ & A & A \\
\hline TSSK6:T989C & rs17851212 & Extragenic & 1,427 & ND & 1.653 & $\mathbf{T}$ & T & $\mathbf{T}$ & $\mathbf{T}$ & $T$ & $\mathbf{T}$ \\
\hline D19S215 & UniSTS:28209 & Extragenic & 3,416 & 0.379 & 3.941 & $1 / 4$ & $1 / 1$ & $1 / 1$ & $1 / 1$ & $1 / 2$ & $3 / 4$ \\
\hline
\end{tabular}

Single allele nomenclature is used for homozygous SNPs, while both alleles are shown for heterozygous SNPs. Single repeat numbers denote homozygous microsatellite repeats, while both repeats are shown for heterozygous ones. Black-shaded: mutation 1623_1624delinsTT. Gray-shaded: distal markers defining and flanking the mutated haplotype. PD, Physical Distance to IL12RB1 expressed in mega-bases (Mb); AF, Allelic Frequency; RF, Recombination Frequency; P1-P6, Patients 1-6; ND, Not Done; im, IL12RB1 intragenic marker (null recombination was assumed); Homoz., Homozygous for mutation 1623_1624delinsTT; Heteroz. Heterozygous for mutation 1623_1624delinsTT; IL12RB1 ex., IL12RB1 exonic; IL12RB1 int., IL12RB1 intronic. 
(A)

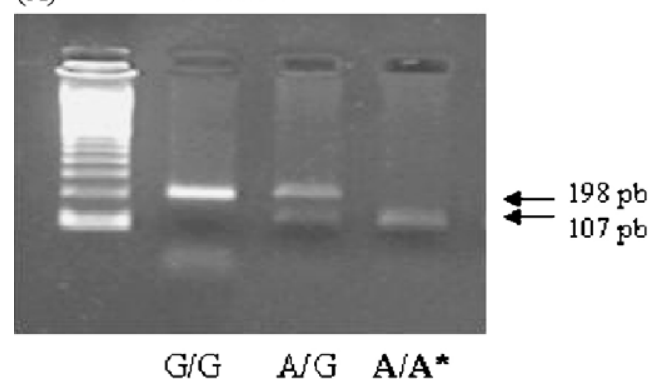

(B)

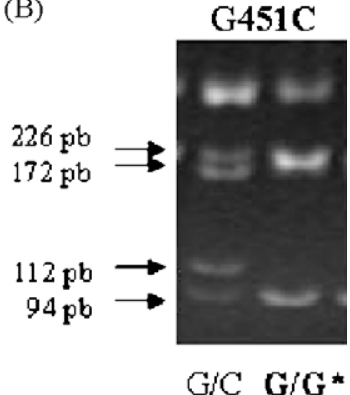

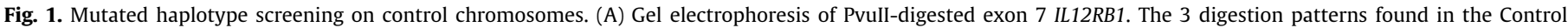

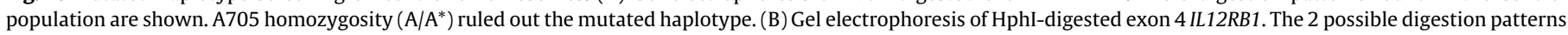
found in the Control population are shown. G451 homozygosity $\left(\mathrm{G} / \mathrm{G}^{*}\right)$ ruled out the mutated haplotype.

interval. Variations on the ancestral allele frequency for D19S1037, arbitrarily set to $1 / 7\left(1 / \mathrm{n}^{\circ}\right.$ of described alleles), only had a slight influence on inference. Specifically, for an allele frequency of 0.5 , the estimation would correspond to 20 generations ( $95 \% \mathrm{CI}, 7-52$ ), while if D19S1037 allele frequency contribution were set to 0 , the inferred number of generations was 19 , same as initial calculations $(95 \% \mathrm{CI}$, 7-50). A basic model assumption for the derived estimations was a phylogeny with star topology (i.e., equal distance to a common ancestor) and so haplotype relatedness would bias computed generation numbers. In an attempt to assess this assumption, age to origin was evaluated for 9 sub-samples, sequentially dropping one chromosome at the time. All estimations rounded the initial calculation of 19 generations with a range of 13 generations (95\% $\mathrm{CI}, 3-40$ ) to 22 generations (95\% CI, 8-60). This relatively small variation suggests that this assumption is valid, though relatedness among some inherited haplotypes cannot be ruled out. In conclusion, the estimation of 19 generations (7-51 with a $95 \%$ confidence) to a common origin for the 1623_1624delinsTT mutation appeared robust.

\section{Discussion}

In the IL12RB1 gene mutation database (http://153.1.44.13/ IL12RB1base/), 24 different mutations affecting 50 individuals from 38 unrelated families are summarized. Mutation 1623_1624delinsTT is described in 7 individuals (5 homozygous and 2 heterozygous) from 4 different families previously reported by Fieschi et al. (2003). This same mutation is even more prevalent among Argentinean IL-12R $\beta 1$ deficient patients: 8-out-of-12 affected alleles (almost 67\%) bear mutation 1623_1624delinsTT. Noteworthy, although all the Argentinean IL-12R $\beta 1$ deficient patients, as well as the Belgian patient studied, belonged to families with European ancestry, their families did not appear to be related.

When identical mutations are detected in apparently unrelated patients, it is important to discriminate whether they have aroused by recurrent mutations (mutational hot spot) or they are identical by descent (mutational founder effect). In this case, the absence of direct repeats, palindromes/quasi-palindromes, polypurine runs, polypyrimidine runs or consensus motifs typically associated with different types of mutational hotspots in the surrounding area argued against a putative mutational hotspot (Krawczak and Cooper, 1993). On the other hand, the detection of a common haplotype spanning 1.45 (distance between C72A and TSSK:T989C, distal markers common to all affected alleles) to $3.51 \mathrm{Mb}$ (distance between D19S1037 and D19S215, first markers to show differences on the affected alleles), strongly supports the mutational founder effect hypothesis.

Many Mendelian disorders demonstrate mutations that can be traced to a founder whose existence can be inferred from the unique chromosomal background on which the mutation occurred
(Zeegers et al., 2004). Founder mutations have been described for several primary immunodeficiencies, including those conferring MSMD. Mutation g.482 + 82_856-854 (a large loss-of-function deletion) and g.315_316insA (a frameshift insertion) in IL12B (IL12p40) are founder mutations associated to increased susceptibility to mycobacteria and salmonella infections arising 700 and 1100 years ago, respectively (Picard et al., 2002; Li et al., 2002; Campbell et al., 2003; Sanchez et al., 2007). In the case of IL-12R $\beta 1$ deficiency due to mutation 1623_1624delinsTT, the founder effect was estimated to occur 475 years ago (95\% CI, 175-1275). To determine so, a slightly modified likelihood method from that originally described by Genin et al. (2004) was applied. The introduced modifications used different parameters to better adjust the intrinsic bias on mutation age-estimation inherent to this type of calculations.

It is noteworthy, that the city of Buenos Aires was funded by Pedro de Mendoza in 1536, approximately at the same time as the estimated arousal of mutation 1623_1624delinsTT. It is tempting to speculate that mutation 1623_1624delinsTT was introduced into the country in the mid-16th century by the Spanish colonization. The current Argentinean population has a genetically heterogeneous ethnic background mainly conferred by waves of European immigrants and their integration to a sparse native population. Following the colonization by the Spaniards in the mid-16th century there was a significant decrease ("bottlenecks") on the native population associated with massive killings and transmitted

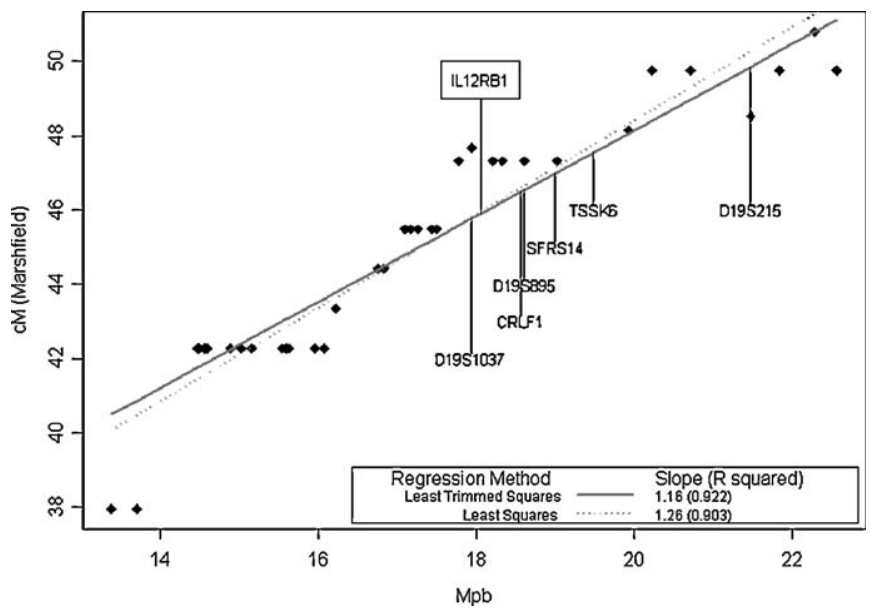

Fig. 2. Extragenic markers recombination fraction estimation. Known distances and linkage for polymorphic sites surrounding IL12RB1 locus (filled rhombi) are shown. Linkage estimation for the extragenic markers indicated on the graph was based on a robust regression method. The slope derived from the least squares method is also despicted, although it shows a poorer fit and a slight bias due to outlying observations. 
diseases (Sanchez et al., 2007; Martínez Marignac et al., 2004; Mulligan et al., 2004; Alfaro et al., 2005; Resano et al., 2007). While mating between the European colonizers and the natives helped to spread the mutation, the reduction on the local population contributed to the mutation enrichment of the genetic pool.

In summary, herein we present strong evidence to support that mutation IL12RB1 1623_1624delinsTT, a rare genomic change, is inherited as a haplotype block with a common founder arousing 475 years ago (95\% CI, 175-1275). This finding represents the first founder effect described on the IL-12/23 receptor complex, critical for controlling mycobacterial as well as salmonella infections, and affecting patients with European ancestry. The reason(s) behind the persistency of this mutation across multiple generations, or whether it confers any kind of selective advantage, has yet to be established.

\section{Acknowledgments}

JY and SDR work is supported by the National Institutes of Health Fogarty International Center, and by the Fogarty International Research Collaboration Award Grant R01TW006644. The authors have no conflict of interests to declare.

\section{Appendix A. Appendix}

\section{A.1. Likelihood correction for mutation}

Beside recombination, haplotype differences can also be explained by mutation. Some formulations from Genin et al. (2004) were applied to account for this ambiguity. Assuming known mutation rates, $\mu_{1}, \ldots, \mu_{x}, \ldots, \mu_{k}$ (for $M_{1}, \ldots, M_{x}, \ldots, M_{\mathrm{k}}$, respectively), the probability that during $n$ generations no marker mutation has occurred from $\Delta$ up to $M_{x}$, denoted $U(x)$, is

$U(x)=\prod_{w=1}^{x}\left(1-\mu_{h}\right)^{n}$

and the probability of mutation at marker $M_{x}$ over $n$ generations, $u(x)$, has the expression

$u(x)=1-\left(1-\mu_{x}\right)^{n}$.

Thus, the likelihood contribution for a chromosome $i$ which shares an ancestral haplotype until marker $M_{x-1}$ and has different allele at marker $M_{x}$ is

$h(x)=U\left(x_{i}-1\right)\left[f\left(x_{i}\right)+\mu_{x_{i}} S\left(x_{i}\right)\right]$,

which contemplates the possibilities that either a recombination occurred between markers $M_{x-1}$ and $M_{x}$ or no recombination took place up to marker $M_{x}$ but a mutation event did happen at $M_{x}$.

Lastly, to account for mutation in the likelihood formulations, the following corrections were applied to equations (1)-(4) in the main text:

$$
\begin{aligned}
& L_{G_{1}}^{\text {mut }}(n)=h(k)^{y}+y U(k) S(k) h(k)^{y-1} \\
& L_{G_{2}}^{\text {mut }}(n)=\prod_{i \in G_{2}} h\left(x_{i}\right) \\
& L_{G_{3}}^{\text {mut }}(n)=[U(k) S(k)]^{z} \\
& L_{G_{4}}^{\text {mut }^{2}}(n)=\prod_{j \in G_{4}} U\left(x_{j}\right) S\left(x_{j}\right)
\end{aligned}
$$

\section{A.2. Likelihood correction for allele frequency}

When polymorphisms of markers are low, an event of recombination with the same allele becomes more plausible. Thereafter, to
Table A1

Primers.

Intragenic markers

SNP

C72A (exon 1)

C202T (exon 3)

G451C (exon 4)

G531A (exon 5)

A705G (exon 7)

C748T (exon 7)

C1081A (exon 9)

T1158C (exon 10)

G1196C (exon 10)

C1213T (exon 10)

C1306T (exon 11)

C1376T (exon 11)

G1637A (exon 13)

\begin{tabular}{|c|c|c|}
\hline Amplicon & & Sequence $\left(5^{\prime} \rightarrow 3^{\prime}\right)$ \\
\hline D19S1037 & $\begin{array}{l}\text { Forward } \\
\text { Reverse }\end{array}$ & $\begin{array}{l}\text { ctg cggagt cag aaa aca gt } \\
\text { atg cag cta tcc ctc att ca }\end{array}$ \\
\hline CRLF1 (exon 2) & $\begin{array}{l}\text { Forward } \\
\text { Reverse }\end{array}$ & $\begin{array}{l}\text { cca act tac ggc caa cat ag } \\
\text { aca atc att aac agc gtc ttt t }\end{array}$ \\
\hline D19S895 & $\begin{array}{l}\text { Forward } \\
\text { Reverse }\end{array}$ & $\begin{array}{l}\text { ttg cag taa cca tgc cac } \\
\text { cca gga cac ttt tgc tag tca c }\end{array}$ \\
\hline SFRS14 (exon 3) & $\begin{array}{l}\text { Forward } \\
\text { Reverse }\end{array}$ & $\begin{array}{l}\text { aac ttt ggc tga ttt cct tac c } \\
\text { tcc agg ctg att gag aaa gag t }\end{array}$ \\
\hline TSSK6 (exon 1) & $\begin{array}{l}\text { Forward } \\
\text { Reverse }\end{array}$ & $\begin{array}{l}\text { cct ggc gga cgg gct gaa ctg } \\
\text { gcg cca tgg ctt aga ccc gag att }\end{array}$ \\
\hline D19S215 & $\begin{array}{l}\text { Forward } \\
\text { Reverse }\end{array}$ & $\begin{array}{l}\text { cat gca tta aaa atg aca act gt } \\
\text { gct ctg can tcc att act ca }\end{array}$ \\
\hline
\end{tabular}

C1783T (exon 15)

G1845C (exon 15)

Extragenic markers

account for hindered crossing-over, a modification to the second order approximation suggested in Genin et al. (2004) was implemented.

Let $p_{x}$ be the frequency of the allele present on the ancestral haplotype at the $M_{x}$ locus. Considering only the possibility of two unnoticed events of recombination, expressions (1)-(4) become:

$$
\begin{aligned}
L^{\text {allele }}(n)= & f(k)^{y}+y p_{k-1} f(k-1) f(k)^{y-1} \\
& +\left(\begin{array}{c}
y \\
2
\end{array}\right) p^{2} f(k-1)^{2} f(k)^{y-2}+y p_{k-1} p_{k-2} f(k-2) f(k)^{y-1} \\
& +y(y-1) p^{2} p_{k-2} f(k-2) f(k-1) f(k)+y S(k) f(k)^{y-1} \\
& +y(y-1) S(k) p_{k-1} f(k-1) f(k)^{y-2} \\
& +y\left(\begin{array}{c}
y-1 \\
2
\end{array}\right) p^{2} S(k) f(k-1)^{2} f(k)^{y-3}+y(y \\
& -1) p_{k-1} p_{k-2} S(k) f(k-2) f(k)^{y-2}+y(y-1)(y \\
& -2) p^{2} p_{k-2} S(k) f(k-2) f(k-1) f(k)
\end{aligned}
$$

- The term with coefficient $\left(\begin{array}{c}y-1 \\ 2\end{array}\right)$ is only computed if $y>2$.

$$
\begin{aligned}
& L^{\text {allele }}(n)=\prod_{i \in G_{2}}\left[f\left(x_{i}\right)+p_{x_{i}-1} f\left(x_{i}-1\right)+p_{x_{i}-1} p_{x_{i}-2} f\left(x_{i}-2\right)\right], \\
& L^{\text {allele }}(n)=\left[S(k)+p_{k} f(k)+p_{k} p_{k-1} f(k-1)\right]^{z}, \\
& L^{\text {allele }}(n)=\prod_{j \in G_{4}}\left[S\left(x_{j}\right)+p_{x_{j}} f\left(x_{j}\right)+p_{x_{j}-1} p_{x_{j}} f\left(x_{j}-1\right)\right] .
\end{aligned}
$$


Note that the only difference to previous formulations from Genin et al. (2004) are the terms for $L_{G 1}(n)$ that contemplate recombination on $M_{k-1}$ and $M_{k-2}$ on two independent events, namely

$y(y-1) p^{2} p_{k-2} f(k-2) f(k-1) f(k)^{y-2}$

and

$y(y-1)(y-2) p^{2} p_{k-2} S(k) f(y-2) f(y-1) f(k)^{y-3}$.

Following the same line of thought that derives in the original expression, the extension includes the probabilities of observing ancestral alleles at $M_{k-1}$ and $M_{k-2}$ after one recombination event on the $(k-2)$ th interval, $p_{k-1} p_{k-2}(k-2)$, and one ancestral allele at $M_{k-1}$ after one recombination event on the $(k-1)$ th interval, $p_{k-1}(k-1)$. The additional terms make a more significant contribution to the final likelihood as $(y-1) p_{k-1}$ increases and $f(k) / f(k-1)$ decreases.

\section{References}

Alfaro, E.L., Pierini, J.E., Gutierrez, N.I., Vullo, C.M., 2005. Genetic structure and admixture in urban populations of the Argentine North-West. Ann. Hum. Biol. 32, 724-737.

Altare, F., Durandy, A., Lammas, D., Emile, J.F., Lamhamedi, S., Le Deist, F., Drysdale, P., Jouanguy, E., Döffinger, R., Bernaudin, F., Jeppsson, O., Gollob, J.A., Meinl, E., Segal, A.W., Fischer, A., Kumararatne, D., Casanova, J.L., 1998. Impairment of mycobacterial immunity in human interleukin-12 receptor deficiency. Science 280, 1432-1435.

Campbell, C., Mitui, M., Eng, L., Coutinho, G., Thorstenson, Y., Gatti, R.A., 2003. ATM mutations on distinct SNP and STR haplotypes in ataxia-telangiectasia patients of differing ethnicities reveal ancestral founder effects. Hum. Mutat. 21, 80-85.

de Jong, R., Altare, F., Haagen, I.A., Elferink, D.G., Boer, T., van Breda Vriesman, P.J., Kabel, P.J., Draaisma, J.M., van Dissel, J.T., Kroon, F.P., Casanova, J.L., Ottenhoff, T.H., 1998. Severe mycobacterial and Salmonella infections in interleukin-12 receptor-deficient patients. Science 280, 1435-1438.

Fieschi, C., Dupuis, S., Catherinot, E., Feinberg, J., Bustamante, J., Breiman, A., Altare, F., Baretto, R., Le Deist, F., Kayal, S., Koch, H., Richter, D., Brezina, M., Aksu, G. Wood, P., Al-Jumaah, S., Raspall, M., Da Silva Duarte, A.J., Tuerlinckx, D., Virelizier, J.L., Fischer, A., Enright, A., Bernhöft, J., Cleary, A.M., Vermylen, C., Rodriguez-Gallego, C., Davies, G., Blütters-Sawatzki, R., Siegrist, C.A., Ehlayel, M.S., Novelli, V., Haas, W.H., Levy, J., Freihorst, J., Al-Hajjar, S., Nadal, D., De Moraes Vasconcelos, D., Jeppsson, O., Kutukculer, N., Frecerova, K., Caragol, I., Lammas, D., Kumararatne, D.S., Abel, L., Casanova, J.L., 2003. Low penetrance, broad resistance, and favorable outcome of interleukin 12 receptor $\beta 1$ deficiency, medical and immunological implications. J. Exp. Med. 197, 527-535.

Fieschi, C., Bosticardo, M., de Beaucoudrey, L., Boisson-Dupuis, S., Feinberg, J., Santos, O.F., Bustamante, J., Levy, J., Candotti, F., Casanova, J.L., 2004. A novel form of complete IL-12/IL-23 receptor beta1 deficiency with cell surfaceexpressed nonfunctional receptors. Blood 104, 2095-2101.
Filipe-Santos, O., Bustamante, J., Chapgier, A., Vogt, G., de Beaucoudrey, L., Feinberg, J., Jouanguy, E., Boisson-Dupuis, S., Fieschi, C., Picard, C., Casanova, J.L., 2006. Inborn errors of IL-12/23- and IFN-gamma-mediated immunity: molecular cellular, and clinical features. Semin. Immunol. 18, 347-361.

Genin, E., Tullio-Pelet, A., Begeot, F., Lyonnet, S., Abel, L., 2004. Estimating the age of rare disease mutations: the example of Triple-A syndrome. J. Med. Genet. 41, 445-449.

Haerynck, F., Holland, S.M., Rosenzweig, S.D., Casanova, J.L., Van daele, S., Schelstraete, P., De Baets, F., 2008. Disseminated Mycobacterium avium infection in a patient with a novel mutation in the interleukin-12 receptor $\beta 1$ chain. J. Pediatr. 153, 721-722.

Hoeve, M.A., de Boer, T., Langenberg, D.M.L., Sanal, O., Verreck, F.A.W., Ottenhoff, T.H.M., 2003. IL-12 receptor deficiency revisited: IL-23-mediated signaling is also impaired in human genetic IL-12 receptor b1 deficiency. Eur. J. Immunol 33, 3393-3397.

Krawczak, M., Cooper, D.N., 1993. Human Gene Mutations. Bios Scientific Publishers, Oxford.

Li, L., Moshous, D., Zhou, Y., Wang, J., Xie, G., Salido, E., Hu, D., de Villartay, J.P. Cowan, M.J., 2002. A founder mutation in Artemis, an SNM1-like protein, causes SCID in Athabascan-speaking Native Americans. J. Immunol. 168, 6323-6329.

Martínez Marignac, V.L., Bertoni, B., Parra, E.J., Bianchi, N.O., 2004. Characterization of admixture in an urban sample from Buenos Aires, Argentina, using uniparentally and biparentally inherited genetic markers. Hum. Biol. 76, 543-557.

Mulligan, C.J., Hunley, K., Cole, S., Long, J.C., 2004. Population genetics, history, and health patterns in Native Americans. Annu. Rev. Genomics Hum. Genet. 5, 295 315.

Nachman, M.W., Crowell, S.L., 2000. Estimate of the mutation rate per nucleotide in humans. Genetics 156, 297-304.

Picard, C., Fieschi, C., Altare, F., Al-Jumaah, S., Al-Hajjar, S., Feinberg, J., Dupuis, S. Soudais, C., Al-Mohsen, I.Z., Génin, E., Lammas, D., Kumararatne, D.S., Leclerc, T. Rafii, A., Frayha, H., Murugasu, B., Wah, L.B., Sinniah, R., Loubser, M., Okamoto, E., Al-Ghonaium, A., Tufenkeji, H., Abel, L., Casanova, J.L., 2002. Inherited interleukin-12 deficiency: IL12B genotype and clinical phenotype of 13 patients from six kindreds. Am. J. Hum. Genet. 70, 336-348.

Resano, M., Esteban, E., González-Pérez, E., Vía, M., Athanasiadis, G., Avena, S., Goicoechea, A., Bartomioli, M., Fernández, V., Cabrera, A., Dejean, C., Carnese, F., Moral, P., 2007. How many populations set foot through the Patagonian door? Genetic composition of the current population of Bahía Blanca (Argentina) based on data from 19 Alu polymorphisms. Am. J. Hum. Biol. 19, 835-877.

Rosenzweig, S.D., Yancoski, J., Bernasconi, A., Krasovec, S., Marciano, B.E., Casimir, L. Berberian, G., Simboli, N., Rousseau, M., Calle, G., 2006. Thirteen years of culture-positive M. bovis-BCG infection in an IL-12Rbeta1 deficient patient: treatment and outcome. J. Infect. 52, e69-e72.

Sanchez, J.J., Monaghan, G., Børsting, C., Norbury, G., Morling, N., Gaspar, H.B., 2007. Carrier frequency of a nonsense mutation in the adenosine deaminase (ADA) gene implies a high incidence of ADA-deficient severe combined immunodeficiency (SCID) in Somalia and a single, common haplotype indicates common ancestry. Ann. Hum. Genet. 71, 336-347.

van de Vosse, E., Ottenhoff, T.H., 2006. Human host genetic factors in mycobacterial and Salmonella infection: lessons from single gene disorders in IL-12/IL-23dependent signaling that affect innate and adaptive immunity. Microbes Infect. 8, 1167-1173.

Weber, J.L., Wong, C., 1993. Mutation of human short tandem repeats. Hum. Mol. Genet. 2, 1123-1128.

Zeegers, M.P., van Poppel, F., Vlietinck, R., Spruijt, L., Ostrer, H., 2004. Founder mutations among the Dutch. Eur. J. Hum. Genet. 12, 591-600. 